\title{
Allogeneic cell-based immunotherapy combined with chemotherapy and targeted therapy in advanced pancreatic cancer with metastases: A case report
}

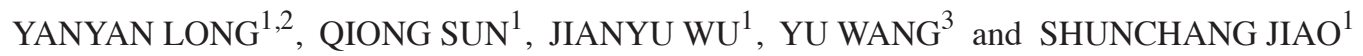 \\ ${ }^{1}$ Department of Medical Oncology, Chinese PLA General Hospital, Beijing 100853; ${ }^{2}$ Medical School of Nankai \\ University, Tianjin 300071; ${ }^{3}$ Beijing ImmunoTech Applied Science Limited, Beijing 101111, P.R. China
}

Received July 23, 2013; Accepted January 30, 2014

DOI: $10.3892 / 01.2014 .1908$

\begin{abstract}
Immunotherapy may be an effective and potentially less toxic treatment for cancer in addition to the traditional therapies. The current study presents a case of advanced pancreatic cancer that was treated with cell-based immunotherapy using expanded activated allogeneic lymphocytes (EAAL $\left.{ }^{*}\right)$ in vitro with cluster of differentiation (CD)3(+) and CD8(+) cytotoxic T lymphocytes, and CD3(-) and CD56(+) natural killer cells as the major effector cells, together with chemotherapy and targeted agents. A 46-year-old female was diagnosed at the Chinese PLA General Hospital (Beijing, China) with stage IV pancreatic cancer with multiple metastases in October 2012. After receiving one cycle of chemotherapy plus nimotuzumab (Nimo), the patient received 14 infusions of EAAL*, which was obtained from a related donor, combined with seven cycles of chemotherapy with gemcitabine plus oxaliplatin and targeted therapy with Nimo. The patient was followed up for eight months. One day prior to the cell infusion, targeted therapy was administered and $48 \mathrm{~h}$ following the cell infusion, chemotherapy was administered. Following this treatment, carbohydrate antigen 19-9 levels decreased from 4,136 U/ml to within the normal ranges, along with the significant regression of the lesions. Occasionally mild upset was observed following the EAAL ${ }^{*}$ transfusion. For the entire combined modality, grade II hematological and gastrointestinal toxicities plus grade I liver function damage and skin rash were identified. The present study demonstrated that combining allogeneic cell-based immunotherapy with conventional therapies is effective and safe, even in patients with end-stage pancreatic
\end{abstract}

Correspondence to: Professor Shunchang Jiao, Department of Medical Oncology, Chinese PLA General Hospital, 28 Fuxing Road, Haidian, Beijing 100853, P.R. China

E-mail: jiaosc@vip.sina.com

Key words: expanded activated allogeneic lymphocytes, expanded activated autologous lymphocytes, pancreatic cancer, adoptive immunotherapy, chemotherapy, nimotuzumab cancer. Therefore, this strategy is recommended for the treatment of similar cases.

\section{Introduction}

Pancreatic ductal adenocarcinoma (PDA), also known as pancreatic cancer, is the fourth leading cause of cancer-related mortality in the United States (1). Survival has not markedly improved despite the routine use of surgery, chemotherapy and radiotherapy. The overall five-year survival rate is $<5 \%$ and the median overall survival is less than six months $(1,2)$. In addition, only $<20 \%$ of patients present with potentially curable localized resectable tumors (3). However, the majority of patients are likely to develop local recurrence or metastasis following surgery. For patients with metastatic disease, PDA is lethal and notoriously difficult to treat and such individuals exhibit a poor median survival of three to six months (4). Over the past decade, gemcitabine (Gem)-based chemotherapy or chemoradiation have been the standard regimen, although, the overall therapeutic efficacy of these methods is considered to be minimal $(5,6)$. In order to improve the current treatment status to achieve greater efficacy and to improve prognosis, novel treatment strategies must be investigated.

Multiple new agents with diverse mechanisms of action in combination with Gem have been previously assessed in randomized clinical trials of pancreatic cancer, with no improvement in outcome observed $(2,7,8)$. To date, the laboratory results of targeted therapies have been significant and only erlotinib, an epidermal growth factor receptor (EGFR)-tyrosine kinase inhibitor, has achieved a modest survival benefit in combination with Gem in a previous phase III clinical trial (9). Nimotuzumab (Nimo) is a humanized monoclonal antibody that recognizes the EGFR extracellular domain. Based on the results of previous phase I/II trials for pancreatic cancer, the recommended dose of Nimo has been established at $200 \mathrm{mg}$ per week. In addition, Nimo is safe and well tolerated, although, the efficacy of monotherapy is minimal. At present, a randomized, placebo-controlled trial of Gem plus Nimo has been initiated, of which the results are of interest (10).

Immunotherapeutic approaches are becoming promising strategies for effectively inducing antitumor immune responses with reduced toxicity (11-13). However, the manner 
in which immunotherapy may be optimally integrated with existing non-immunological therapies for optimal synergy remains to be elucidated (14). In addition, an approach should be established to arrange the order of the various combination treatments, including immunotherapy, chemotherapy and monoclonal antibodies (15-18).

Expanded activated autologous lymphocyte (EAAL) therapy with cluster of differentiation (CD)3(+) and CD8(+) cytotoxic T lymphocytes, and CD3(-) and CD56(+) natural killer cells as the major effector cells is a type of adoptive cell therapy. It has previously been shown that EAAL therapy has the ability to enrich potential antitumor responses and that it is safe for early- and late-stage cancer patients $(19,20)$. A randomized trial sponsored by Takayama et al (21) demonstrated that adoptive immunotherapy lowers postsurgical recurrence rates of hepatocellular carcinoma with significantly longer recurrence-free $(\mathrm{P}=0.01)$ and disease-specific $(\mathrm{P}=0.04)$ survival than those of the control group. Expanded activated allogeneic lymphocyte (EAAL*) therapy is a type of EAAL therapy with infusion lymphocytes, which are obtained from a human leukocyte antigen (HLA)-matched related donor rather than from the patients themselves.

The present study reports the eight-month follow-up of a patient with advanced pancreatic cancer with multiple metastases. The patient was treated with EAAL* therapy obtained from a related donor in addition to conventional chemotherapy with Gem and oxaliplatin (L-OHP) plus targeted therapy with Nimo. Written informed consent was obtained from the family of the patient.

\section{Case report}

A 46-year-old female presented to with cough and expectoration with no apparent cause in October 2012 at the local doctor. Positron emission tomography (PET)/computed tomography (CT) and biopsy revealed a PDA involved in the body of the pancreas with multiple metastases to the lungs, liver and abdominal lymph nodes. The carbohydrate antigen (CA) 19-9 value was 3,318 U/ml at diagnosis. Prior to the cell-based immunotherapy, the patient received one cycle of intravenous chemotherapy with $1,800 \mathrm{mg} \mathrm{Gem}\left(1,000 \mathrm{mg} / \mathrm{m}^{2}\right.$ i.v. on days one and eight, every 21 days) and $150 \mathrm{mg}$ L-OHP ( $85 \mathrm{mg} / \mathrm{m}^{2}$ i.v. on day one, every 21 days), and targeted therapy with $200 \mathrm{mg}$ Nimo (i.v. on day seven, every seven days). The immunotherapy was subsequently initiated. At diagnosis, the tumor load of the patient was considered to be large, due to multiple metastases, and the patient had relatively weak immunity, thus, the EAAL* therapy was designed (Beijing ImmunoTech Applied Science Ltd., Beijing, China). Written informed consent was obtained and the patient's HLA genotype was matched with that of a related donor, peripheral blood was collected from the related donor in heparin tubes and transported to the laboratory under cold conditions.

Activated lymphocytes using anti-CD3 monoclonal antibody and interleukin-2 were generated as described previously (22). Briefly, 20-100 ml of peripheral blood was collected from the related donor and peripheral blood mononuclear cells (PBMCs) were isolated by Ficoll-Hypaque gravity centrifugation (ALLEGRA X-12, Beckman Coulter, Miani, FL, USA) at $400 \mathrm{x}$ g. The isolated PBMCs were washed and resuspended in serum-free medium (IMSF 100; Immunotech, London, UK) supplemented with $700 \mathrm{U} / \mathrm{ml}$ of interleukin (IL)-2 (CCBIO, Changchun, China). The PBMC suspension was placed in a flask coated with immobilized anti-CD3 antibody (eBioscience, San Diego, CA, USA)and incubated for one week. The lymphocyte suspension was transferred to a gas-permeable bag to allow the lymphocytes to grow for two more weeks. The activated lymphocytes were subsequently harvested, filtered through $100-\mu \mathrm{m}$ membranes and resuspended in $100 \mathrm{ml}$ of normal saline containing $1 \%$ human serum albumin for the intravenous infusion. Prior to cell transplantation, the cells were assessed for endotoxin levels using a Limulus Amebocyte Lysate kit (Associates of Cape Cod, Inc., Falmouth, MA, USA). The average cell count following the in vitro expansion was $2.04-5.18 \times 10^{9}$ cells $/ 100 \mathrm{ml}$. Therefore, the patient was administrated 100 or $200 \mathrm{ml}$ (large dose) of these activated lymphocytes up to 14 times a week or every other week. For each infusion, one part of these cells was recovered, activated and expanded for two weeks and transferred into the patient as previously described.

Overall, the patient received 14 infusions of EAAL*, with an overall cell count of $7.414 \times 10^{10}$, in combination with seven cycles of chemotherapy plus targeted therapy. The initial four infusions of EAAL* were at doses of $100 \mathrm{ml}$ and were followed by doses of $200 \mathrm{ml}$. The specific regimen was as follows: EAAL*, $100 \mathrm{ml} / 200 \mathrm{ml}$ i.v. on days 2 and 9; Gem, $1,800 \mathrm{mg}$ i.v. on days 4 and 11; L-OHP, $150 \mathrm{mg}$ i.v. on day 4; and Nimo, $200 \mathrm{mg}$ i.v. on days 1, 8 and 15, every 21 days. Of note, during the last two cycles of chemotherapy, the dosage of Gem was reduced to $1,600 \mathrm{mg}$ and that of L-OPH was reduced to $125 \mathrm{mg}$, due to concern regarding the cumulative toxicities, although, the patient tolerated the treatments well exhibiting only grade II adverse effects.

Responses were evaluated according to the Response Evaluation Criteria in Solid Tumors (RECIST) and the toxic effects were assessed using the National Cancer Institute Common Toxicity Criteria, version $3.0(23,24)$. The responses were recorded during and following each infusion and the final follow-up was in July 2013.

Following two infusions of EAAL* in combination with one cycle of chemotherapy plus targeted therapy, the patient's response was evaluated. A CT scan in December 2012 revealed that lesions in the pancreas, liver, lungs and abdominal lymph nodes showed slight shrinkage. In addition, the CA 19-9 levels had decreased from 4,136 U/ml (prior to treatment) to $758.50 \mathrm{U} / \mathrm{ml}$ (Fig. 1). The status of the patient was stable disease according to the RECIST criteria.

The patient continued with six infusions of EAAL* combined with three cycles of chemotherapy plus targeted therapy. Responses were evaluated by CT scan, which revealed that the metastatic lesions in the lungs and liver were significantly reduced, with lesions in the pancreas being slightly reduced. In addition, the CA 19-9 levels decreased to 113.6 U/ml (Fig. 1) and partial remission (PR) was achieved.

Finally, the patient received a further six infusions of EAAL* at large doses $(200 \mathrm{ml})$ together with three cycles of chemotherapy plus targeted therapy. PET/CT was performed to evaluate the responses and showed that almost all the metabolic values of the deposits had markedly decreased or even disappeared; in addition, the size of all the lesions had 


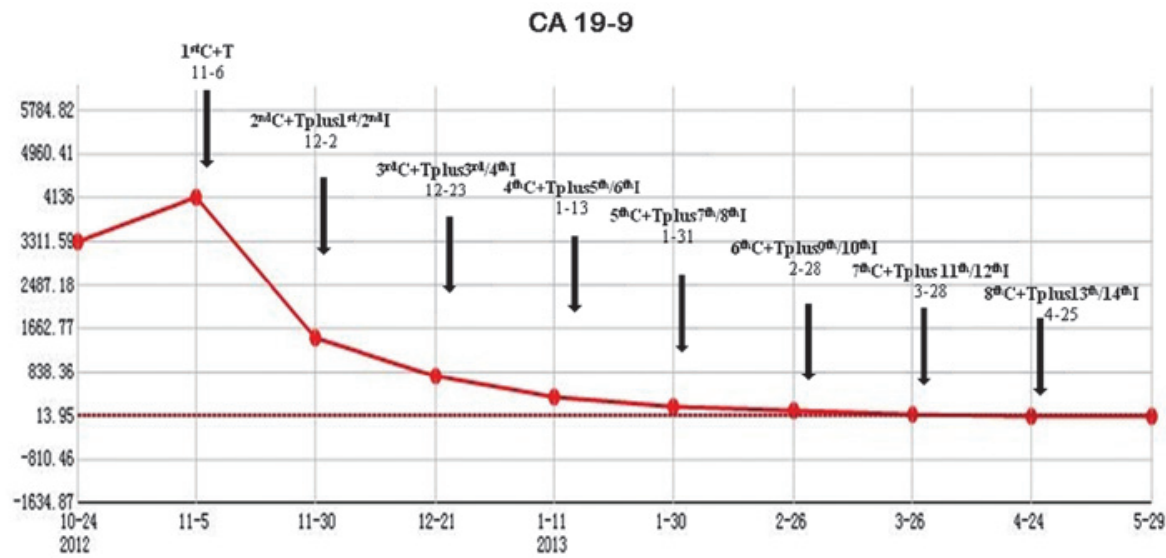

Figure 1. Curve demonstrating the decrease in CA 19-9 values during the whole course of treatment, with eight cycles of chemotherapy plus targeted therapy combined with 14 infusions of EAAL therapy. CA 19-9, carbohydrate antigen 19-9; EAAL*, expanded activated allogeneic lymphocytes; C, chemotherapy with gemcitabine and oxaliplatin; T, targeted therapy with nimotuzumab; I, EAAL"-based immunotherapy.

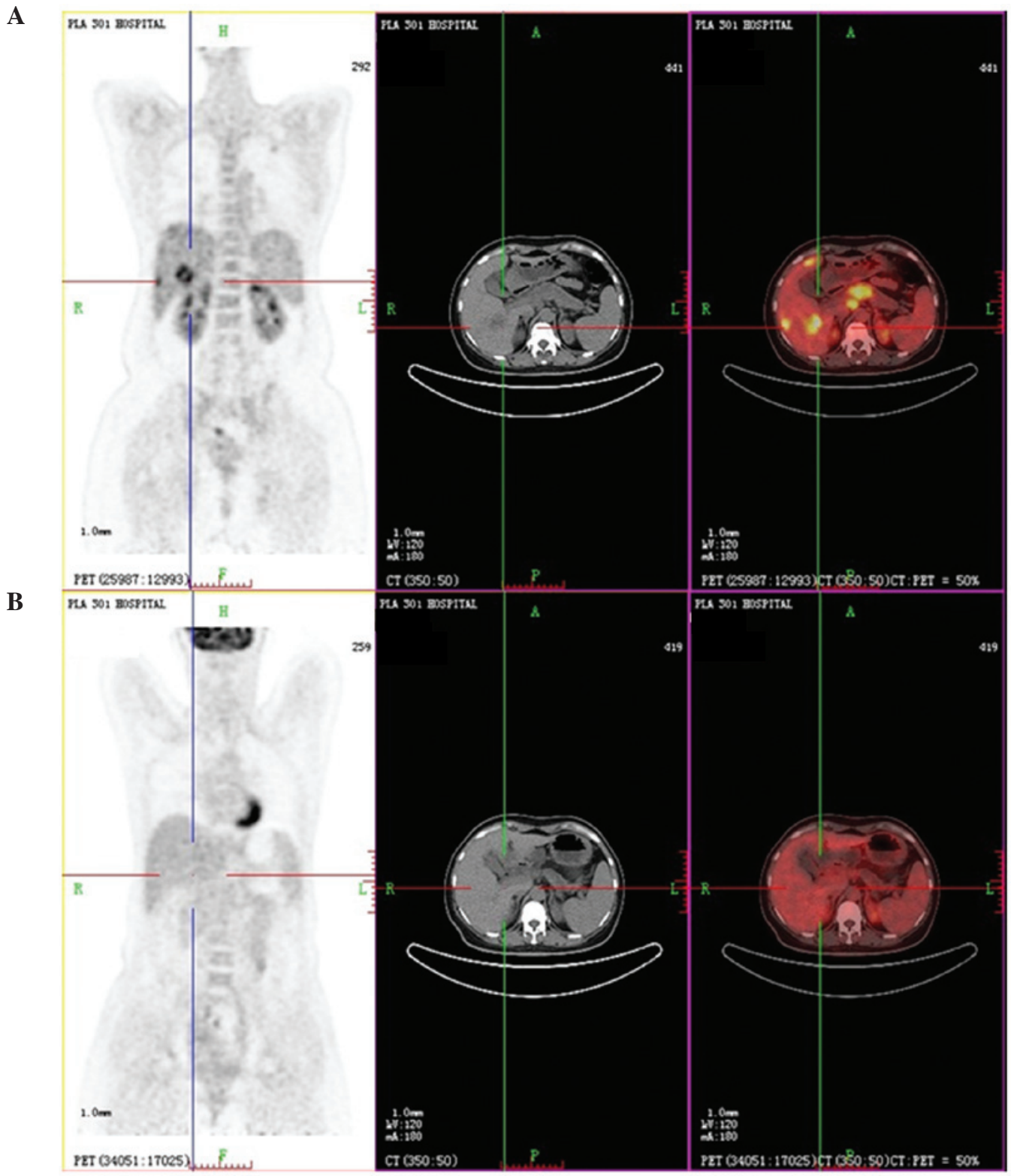

Figure 2. Positron emission tomography/computed tomography of the patient prior to the first cycle of chemotherapy plus targeted therapy on (A) Oct 29th, 2012 and (B) following the eighth cycle of chemotherapy plus targeted therapy combined with 14 infusions of expanded activated allogeneic lymphocyte therapy on May 28th, 2013. The metabolic values of the deposits in the pancreas, liver and abdominal lymph nodes decreased markedly and even disappeared. In addition, the deposits in the lungs decreased significantly, along with the size of all the lesions compared with the lesion sizes prior to treatment. 
markedly decreased (Fig. 2). The CA 19-9 levels decreased to $24.09 \mathrm{U} / \mathrm{ml}$ (normal range, 0.1-37 U/ml; Fig. 1) and PR and near complete remission (nCR) were achieved.

The last follow-up in July 2013 revealed a static non-progressive disease with a progression-free survival (PFS) of eight months and demonstrated that all of the parameters, including the tumor markers, were within their normal ranges.

The patient showed an improved quality of life without a cough or expectoration. Following all 14 infusions of EAAL ${ }^{*}$, with an overall cell count of $7.414 \times 10^{10}$, mild upset was occasionally identified following large-dosage lymphocyte transfusions without other severe adverse effects. For the whole combined modality, the most serious toxicities observed were grade II hematological and gastrointestinal toxicities, in addition to grade I liver function damage and a skin rash.

\section{Discussion}

The current study presents a patient with stage IV pancreatic cancer with multiple metastases for whom curative surgery was not an option at diagnosis. A novel therapeutic strategy was administered to the patient, which included several infusions of EAAL* together with Gem-based chemotherapy and Nimo-based targeted therapy. Notably, the strategy achieved an ideal and rare antitumor responses, PR and nCR. The CA 19-9 levels decreased from 4,136 $\mathrm{U} / \mathrm{ml}$ to within the normal ranges. In addition, significant regression of the lesions was observed. The eight-month follow-up showed a prolonged static non-progressive disease with a PFS of seven months, which far exceeded the predictions of a previous study (4). The patient benefited from the individualized treatment and multimodality therapy, which is consistent with the previous observations that immunotherapy combined with other non-immunological therapies moderately enriches the potential antitumor responses through the mechanism(s) by which these modalities are synergized. However, these mechanisms are not fully understood (17).

Of note, the order of administration for the combined therapeutic approach is a critical factor that affects the therapeutic outcome. Therefore, establishing the order of administration to maximize the efficacy and guarantee safety is a significant problem that remains unsolved. Zhang et al (16) hypothesized that timely immune modification of chemotherapy-activated antitumor immunity results in an enhanced antitumor immune response and complete tumor eradication. In accordance with this, chemotherapy is commonly administered prior to autologous cell-based immunotherapy in clinical practice. However, for safety considerations, the EAAL" was administered prior to chemotherapy in the current study. It was hypothesized that allogeneic cell infusion may be relatively unsafe compared with infusions of autologous lymphocytes, as it may lead to rejection or unknown adverse effects. Therefore, the current patient received chemotherapy $48 \mathrm{~h}$ following the allogeneic cell infusion since toxic chemotherapy agents are likely to eventually remove foreign cells. The present study showed that EAAL ${ }^{*}$ therapy was safe and the order of administration was effective.

To date, the patient has achieved nCR with marked regression of the deposits. Immunotherapy has been planned as a maintenance treatment to lower the local recurrence and metastasis rates, and eradicate any minimal residual disease. In addition, follow-up treatment and prognosis will be tracked for subsequent studies.

In conclusion, the current study showed that this type of combined therapy is effective and safe, therefore, we recommend that this strategy is considered for the treatment of similar cases.

\section{Acknowledgements}

The authors would like to thank the patient and family for their support and cooperation with the present study.

\section{References}

1. Jemal A, Siegel R, Ward E, et al: Cancer statistics, 2008. CA Cancer J Clin 58: 71-96, 2008.

2. Li D, Xie K, Wolff R and Abbruzzese JL: Pancreatic cancer. Lancet 363: 1049-1057, 2004.

3. Wong $\mathrm{HH}$ and Lemoine NR: Pancreatic cancer: molecular pathogenesis and new therapeutic targets. Nat Rev Gastroenterol Hepatol 6: 412-422, 2009.

4. Pancreatric Section, British Society of Gastroenterology; Pancreatic Society of Great Britain and Ireland; Association of Upper Gastrointestinal Surgeons of Great Britain and Ireland; Royal College of Pathologists; Special Interest Group for Gastro-Intestinal Radiology: Guidelines for the management of patients with pancreatic cancer periampullary and ampullary carcinomas. Gut 54 (Suppl 5): v1-v16, 2005.

5. Burris HA III, Moore MJ, Andersen J, et al: Improvements in survival and clinical benefit with gemcitabine as first-line therapy for patients with advanced pancreas cancer: a randomized trial. J Clin Oncol 15: 2403-2413, 1997.

6. Hidalgo M: Pancreatic cancer. N Engl J Med 362: 1605-1617, 2010.

7. Sultana A, Smith CT, Cunningham D, Starling N, Neoptolemos JP and Ghaneh P: Meta-analyses of chemotherapy for locally advanced and metastatic pancreatic cancer. J Clin Oncol 25: 2607-2615, 2007.

8. Heinemann V, Boeck S, Hinke A, Labianca R and Louvet C: Meta-analysis of randomized trials: evaluation of benefit from gemcitabine-based combination chemotherapy applied in advanced pancreatic cancer. BMC Cancer 8: 82, 2008.

9. Moore MJ, Goldstein D, Hamm J, et al: Erlotinib plus gemcitabine compared with gemcitabine alone in patients with advanced pancreatic cancer: a phase III trial of the National Cancer Institute of Canada Clinical Trials Group. J Clin Oncol 25: 1960-1966, 2007.

10. Strumberg D, Schultheis B, Scheulen ME, et al: Phase II study of nimotuzumab, a humanized monoclonal anti-epidermal growth factor receptor (EGFR) antibody, in patients with locally advanced or metastatic pancreatic cancer. Invest New Drugs 30: 1138-1143, 2012

11. Vonderheide RH, Bajor DL, Winograd R, Evans RA, Bayne LJ and Beatty GL: CD40 immunotherapy for pancreatic cancer. Cancer Immunol Immunother 62: 949-954, 2013.

12. Sekine T, Takayama T, Konomi Y and Kakizoe T: Prevention of cancer recurrence by infusion of activated autologous lymphocytes. Hum Cell 7: 121-124, 1994 (In Japanese).

13. Dudley ME and Rosenberg SA: Adoptive-cell-transfer therapy for the treatment of patients with cancer. Nat Rev Cancer 3: 666-675, 2003.

14. Ostrand-Rosenberg S: Looking to the future of cancer immunotherapy: many questions to answer and many therapeutic opportunities. Cancer Immunol Immunother 62: 1-2, 2013.

15. Baxevanis CN, Perez SA and Papamichail M: Combinatorial treatments including vaccines, chemotherapy and monoclonal antibodies for cancer therapy. Cancer Immunol Immunother 58: 317-324, 2009.

16. Zhang L, Feng D, Yu LX, Tsung K and Norton JA: Preexisting antitumor immunity augments the antitumor effects of chemotherapy. Cancer Immunol Immunother 62: 1061-1071, 2013.

17. Ramakrishnan R and Gabrilovich DI: Novel mechanism of synergistic effects of conventional chemotherapy and immune therapy of cancer. Cancer Immunol Immunother 62: 405-410, 2013. 
18. Mitchell MS: Combining chemotherapy with biological response modifiers in treatment of cancer. J Natl Cancer Inst 80: 1445-1450, 1988.

19. Sekine T, Shiraiwa H, Yamazaki T, Tobisu K and Kakizoe T: A feasible method for expansion of peripheral blood lymphocytes by culture with immobilized anti-CD3 monoclonal antibody and interleukin-2 for use in adoptive immunotherapy of cancer patients. Biomed Pharmacother 47: 73-78, 1993.

20. Sun Z, Shi L, Zhang H, et al: Immune modulation and safety profile of adoptive immunotherapy using expanded autologous activated lymphocytes against advanced cancer. Clin Immunol 138: 23-32, 2011.

21. Takayama T, Sekine T, Makuuchi M, et al: Adoptive immunotherapy to lower postsurgical recurrence rates of hepatocellular carcinoma: a randomised trial. Lancet 356: 802-807, 2000.
22. Schmidt-Wolf IG, Negrin RS, Kiem HP, Blume KG and Weissman IL: Use of a SCID mouse/human lymphoma model to evaluate cytokine-induced killer cells with potent antitumor cell activity. J Exp Med 174: 139-149, 1991.

23. Therasse P, Arbuck SG, Eisenhauer EA, et al: New guidelines to evaluate the response to treatment in solid tumors. European Organization for Research and Treatment of Cancer, National Cancer Institute of the United States, National Cancer Institute of Canada. J Natl Cancer Inst 92: 205-216, 2000.

24. Holmboe L, Andersen AM, Mørkrid L, Slørdal L and Hall KS: High dose methotrexate chemotherapy: pharmacokinetics, folate and toxicity in osteosarcoma patients. Br J Clin Pharmacol 73: 106-114, 2012. 